\title{
LIFTING CONSTRUCTION OF NON-LINEAR WAVELET TRANSFORMS
}

\author{
Roger L. Claypoole, Jr. and Richard G. Baraniuk \\ Robert D. Nowak \\ Dept. of Electrical and Computer Engineering \\ Rice University \\ Houston, TX 77005 \\ Dept. of Electrical Engineering \\ Michigan State University \\ East Lansing, MI 48824
}

\begin{abstract}
This paper analyzes non-linear wavelet transforms using the lifting construction. The lifting construction exploits a spatialdomain, prediction-error interpretation of the wavelet transform and provides a powerlul framework for designing customized transforms. We use the lifting construction to better understand the performance of wavelet transforms that utilize median and Volterra filters.
\end{abstract}

\section{INTRODUCTION}

The 1-d discrete wavelet transform (DWT) represents a real-valued discrete-time signal in terms of shifts and dilations of a low-pass scaling function and a bandpass wavelet function [1]. The DWT decomposition is multiscale: it consists of a set of scaling coefficients $c^{0}[n]$, which represent coarse signal information at scale $j=0$, and a set of wavelet coefficients $d^{j}[n]$, which represent detail information at scales $j=1,2, \ldots, J$. The forward DWT has an efficient implementation in terms of a recursive multirate filterbank based around a low-pass filter $h$ and high-pass filter $g$. The inverse DWT employs an inverse filterbank with low-pass filter $\tilde{h}$ and high-pass filter $\widetilde{g}$. For special choices of $h$ and $g$, we have $\widetilde{h}=h$ and $\widetilde{g}=g$, and the underlying wavelet and scaling functions form an orthonormal signal basis. Otherwise, these functions form a biorthogonal basis [1].

The economy of the wavelet transform stems from the fact that the DWT tends to compress real-world signals into just a few coefficients of large magnitude. Compression follows from the "vanishing moments" property of wavelets, which guarantees that the wavelet coefficients of low-order polynomial signals are zero [1]. Thus, if a signal is exactly polynomial, it can be completely described using scaling coefficients alone. In more realistic situations, the signal will not be polynomial, but may be well-approximated by a piecewise polynomial function. Because wavelet functions also have localized support, most of the wavelet coefficients of such a signal will be zero except those corresponding to wavelets having support near the breakpoints of the polynomial segments.

It is fruitful to view the DWT as a prediction-error decomposition. The scaling coefficients at a given scale $(j)$ are "predictors" for the data at the next higher resolution or scale $(j-1)$. The wavelet coefficients are simply the "prediction errors" between the scaling coefficients and the higher resolution data that they are attempting to predict. This interpretation has led to a new framework for DWT design known as the lifting scheme [2].

In this paper we use lifting to design new non-linear DWTs based on non-linear median and Volterra filters. First, in Section

Supported by NSF, grant nos. MIP-9457438 and MIP-9701692, and DARPA through AFOSR grant no. F49620-97-1-0513.

Email: clayporl@ rice.edu, richb@rice.edu, nowak@egr.msu.edu

Web: www.dsp.rice.edu, www.egr.msu.edu
2, we review the basic lifting construction. Then, in Section 3 , we explain how various non-linear wavelet transforms are implemented via lifting. In Section 4, we judge the quality of the new transforms using numerical simulations. We close in Section 5 with concluding remarks and plans for future work.

\section{THE LIFTING CONCEPT}

Lifting, a space-domain construction of biorthogonal wavelets developed by Sweldens [2], consists of the iteration of the following three basic operations (see Figure 1):

Split: Divide the original data into two disjoint subsets. For example, we split the original data set $x[n]$ into $x_{e}[n]=$ $x[2 n]$, the even indexed points, and $x_{o}[n]=x[2 n+1]$, the odd indexed points.

Predict: Generate the wavelet coefficients $d[n]$ as the error in predicting $x_{o}[n]$ from $x_{e}[n]$ using prediction operator $\mathcal{P}$ :

$$
d[n]=x_{o}[n]-\mathcal{P}\left(x_{e}[n]\right)
$$

Update: Combine $x_{e}[n]$ and $d[n]$ to obtain scaling cuefficients $c[n]$ that represent a coarse approximation to the original signal $x[n]$. This is accomplished by applying an update operator $\mathcal{U}$ to the wavelet coefficients and adding to $x_{e}[n]$ :

$$
c[n]=x_{e}[n]+\mathcal{U}(d[n]) .
$$

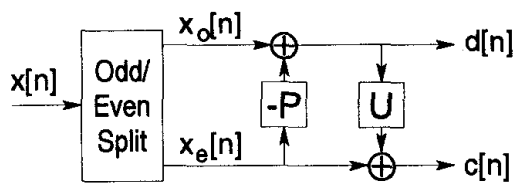

Figure 1: Lifting stage: Split, Predict, Update.

These three steps form a lifting stage. Iteration of the lifting stage on the output $c[n]$ creates the complete set of DWT scaling and wavelet coefficients $c^{j}[n]$ and $d^{j}[n] .^{1}$

The lifting steps are easily inverted, even if $\mathcal{P}$ and $\mathcal{U}$ are nonlinear or space-varying. Rearranging (1) and (2), we have

$$
x_{e}[n]=c[n]-\mathcal{U}(d[n]), \quad x_{o}[n]=d[n]+\mathcal{P}\left(x_{e}[n]\right) .
$$

\subsection{Linear Predictor Design}

Typically, the prediction operator $\mathcal{P}$ is a linear shift-invariant filter, with $z$-transform $P(z)$. In Figure 2, we illustrate a symmetric, $N=4$ point predictor $P(z)=p_{1} z^{-1}+p_{2}+p_{3} z+p_{4} z^{2}$. By tracing the contribution of $x_{e}[n]$ and $x_{o}[n]$ through the tree to the

\footnotetext{
${ }^{1}$ In fact, all wavelet transforms can be factored into a series of lifting stages (with perhaps multiple predicts and updates per stage)[3].
} 
point $d[n]$, we can find the equivalent filter that would be applied to the original data $x[n]$. In vector form, we have

$$
\mathbf{g}=\left[-p_{1}, 0,-p_{2}, 1,-p_{3}, 0,-p_{4}\right]^{T} .
$$

(Note the zeros at the positions corresponding to odd points in the original data, except for the 1 in the center.)

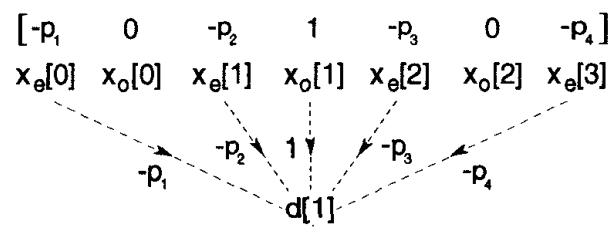

Figure 2: Linear prediction filtering. An $N=4$ point linear prediction filter $P(z)$ yields the prediction vector $\mathrm{g}$ shown across the top.

Recall that the goal of the prediction step is to eliminate all low-order polynomials from $x[n]$ in creating the wavelet coefficients. For a linear predictor, this is easily accomplished by the following simple procedure. Form the $N \times(2 N-1)$ matrix $\mathrm{V}$ whose rows are the monomial signals: $[\mathrm{V}]_{m, n}=n^{m}$, $n=-(N-1), \ldots,(N-1), m=0,1, \ldots, N-1$. (We make the convention $0^{\circ}=1$.) Now, for the predictor to suppress all polynomials up to $N-1$ st order, we require that

$$
\mathrm{Vg}=\mathbf{0} \text {. }
$$

This set of linear equations is readily solved, since $\mathbf{V}$ forms the first $N$ rows of a Vandermonde matrix, which is always invertible. Upon recognizing that the solution lives in an $N$-dimensional subspace, we can rewrite (5) in a simpler form in terms of a new $N \times N$ matrix $V^{\diamond}$ and $\mathbf{p}$ the vector of coefficients of the prediction filter $P(z)$

$$
\mathbf{V}^{\diamond} \mathbf{p}=[1,0, \ldots, 0]^{T} \text {. }
$$

\subsection{Linear Update Design}

The (linear) update filter $U(z)$ creates $c[n]$ by updating each $x_{e}[n]$ with the nearest $\widetilde{N}$ wavelet coefficients $d[n]$ from either side. The update order $\widetilde{N}$ can be chosen independently of $N$; however, the prediction coefficients $p_{k}$ must be fixed prior to determining the update filter in the standard lifting programme.

In Figure 3, we trace the contribution of the original $x_{e}[n]$ and $x_{o}[n]$ to each $c[n]$ for an $N=2$ point predict followed by an $\tilde{N}=4$ point update with $U(z)=u_{1} z^{-2}+u_{2} z^{-1}+u_{3}+u_{4} z$. In vector form, we have the equivalent filter $h$ at the top of the Figure. Note that $h$ is a function of both the update coefficients $u_{k}$ and the prediction coefficients $p_{k}$.

$$
\begin{aligned}
& {\left[-p_{1} u_{1}, u_{1},-p_{1} u_{2}-p_{2} u_{1}, u_{2}, 1-p_{2} u_{2}-p_{1} u_{3}, u_{3},-p_{2} u_{3}-p_{1} u_{4}, u_{4},-p_{2} u_{4}\right]} \\
& x_{e}[0] \quad x_{0}[0] \quad x_{e}[1] \quad x_{0}[1] \quad x_{e}[2] \quad x_{0}[2] \quad x_{e}[3] \quad x_{0}[3] \quad x_{e}[4]
\end{aligned}
$$

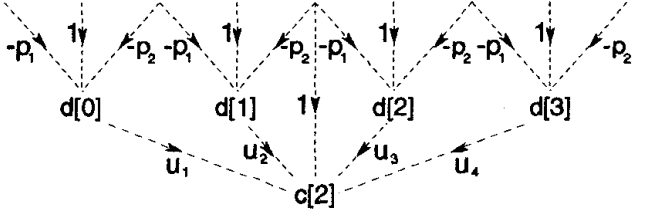

Figure 3: Linear update filtering. An $N=2$ point linear predict followed by an $\widetilde{N}=4$ point linear update yields the update vector $\mathrm{h}$ shown across the top.
The update filter vector $\mathbf{h}$ should pass low-order polynomials into $c[n]$ while attenuating high-order polynomials. This is equivalent to designing the mirror update filter vector $\widetilde{\mathbf{g}}$ (defined as $\left.\widetilde{g}_{n}=(-1)^{n} h_{n}\right)$ to suppress low-order polynomials. For the example in Figure 3, we have

$$
\begin{aligned}
\widetilde{\mathbf{g}}= & {\left[-p_{1} u_{1},-u_{1},\left(-p_{1} u_{2}-p_{2} u_{1}\right),-u_{2},\left(1-p_{2} u_{2}-p_{1} u_{3}\right),\right.} \\
& \left.-u_{3},\left(-p_{2} u_{3}-p_{1} u_{4}\right),-u_{4},-p_{2} u_{4}\right]^{T} .
\end{aligned}
$$

Since the $N=2$ prediction coefficients are already determined, there are $\widetilde{N}=4$ unknowns (the update coefficients $u_{k}$ ) in $\widetilde{\mathbf{g}}$. Solution of $\mathbf{V} \widetilde{\mathbf{g}}=\mathbf{0}$ as in (5) yields the update coefficients.

In summary, we design the prediction step to eliminate the low-order polynomial signal structure, leaving only the high-order details. We design the update to preserve the low-order polynomial signal structure at the next coarser scale. ${ }^{2}$

\section{NON-LINEAR TRANSFORMS VIA LIFTING}

The lifting approach to wavelet design gives us a great deal of flexibility. In principle, we can use any linear, non-linear, or spacevarying predictor and update; the lifting construction ensures that the resulting transform is invertible. We now investigate the capabilities of the lifting approach for designing DWTs based on median and Volterra filters. The motivation behind these transforms is that better prediction should lead to a more efficient signal representation. Since the compression abilities of signal transformations are key to successful signal processing algorithms [4], these new non-linear transforms have the potential to improve transformbased processing.

\subsection{Median Filtering}

Median filtering can be interpreted as adaptive filtering; at each point a linear filter is chosen from a specific family.

Consider the application of an $N$ point median filter. If the median value of the data set is in the $i^{\text {th }}$ position, then median filtering is equivalent to applying a linear filter $e_{i}$, with $e_{i}$ all zero except for $a 1$ in the $i^{\text {th }}$ position. Thus, median filtering is adaptive linear filtering, with each filter chosen from the family of $e_{i}, i=$ $1, \cdots, N$.

Lifting permits us to median filter in the prediction step and then follow this operation by an adaptive update step to preserve the low-pass interpretation of the scaling coefficients $c[n]$. First, compute the wavelet coefficients $d[n]$ using the median filter. For each $n$, we remember which $e_{i}$ was utilized. Then, a tree can be constructed to trace each scaling coefficient up to the the original data $x[n]$, as shown in Figure 4. For each $d[n]$ used to lift the coarse coefficient, we have a contribution from only two members of the original data set. This provides an update vector as described in Section 2.2. We can then apply the appropriate set of linear constraints to solve for the update filter coefficients $u_{i}$.

Each scaling coefficient $c[n]$ is constructed as a low-order polynomial approximation to the original data. A new set of update filter coefficients must be found for each $n$ to ensure each $c[n]$ has a valid polynomial interpretation. Thus, despite the application of the non-linear median filter in the predict step, the update filter coefficients $u_{i}$ adapt to ensure the scaling coefficients satisfy linear polynomial constraints and are a low-pass representation of the original data set. We can now iterate on these coefficients to maintain the recursive, multiscale properties of the wavelet transform.

\footnotetext{
${ }^{2}$ To normalize the energy of the underlying scaling and wavelet functions, we actually output $2^{-1 / 2} d[n]$ and $2^{1 / 2} c[n]$ from the lifting stage.
} 


$$
\left.\begin{array}{lllllllll}
0 & u_{1} & -u_{1} & u_{2} & 1 & u_{3} & -u_{2}-u_{3}-u_{4} & u_{4} & 0
\end{array}\right]
$$

Figure 4: Linear update ilitering. A median filter predict followed by an $\tilde{N}=4$ point linear update yields the update vector $h$ shown across the top.

If we desire an update filter of length $\widetilde{N}$, we must generate $\tilde{N}$ update constraints. If $\tilde{N}=1$, then the solution is $u=1 / 2$ for any choice of median filrer and any data. This is the median filter multiscale transform presented in [5], which can now be viewed as optimal in the sense of polynomial approximation.

For $\tilde{N}>1$, we typically use all the free variables to satisfy polynomial constraints. However, due to the (possibly) overlapping nature of the median filter choices, these constraints may be incompatible, and the resulting matrix $\mathbf{V}^{\circ}$ will not be invertible. In this case, we can incorporate the first two polynomial constraints, and use the remaining free variables to minimize the energy of the update filter. This keeps the filter from becoming highly asymmetrical, even when the median filter choices are greatly overlapped.

A final type of median based transform uses a median filter in the update step. Using our adaptive-filter interpretation of the median filter, we know that a median update filter will choose just one detail coefficient to update each coarse coefficient, as demonstrated in Figure 5. Regardless of the median choices (for the prediction or the update median filter), the choice of $u=1 / 2$ satisfies the $0^{\text {th }}$ polynomial constraint. This double-median transform is useful for preventing large detail coefficients from corrupting the coarse coefficients at the next scale.

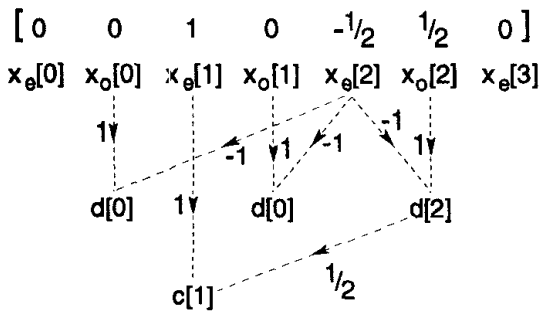

Figure 5: Median filter predict followed by median filter update. The update vector $h$ shown across the top satisfies the $0^{\text {th }}$ polynomial constraint regardless of median choices.

\subsection{Volterra Filtering}

Generally in signal and image processing, the optimal predictor is non-linear (the exception being Gaussian processes). Therefore, it is of interest to consider non-linear predictors. To keep things manageable, the class of Volterra (polynomial) predictors [6] is a reasonable extension of the standard linear predictor. The output of a Volterra filter is a polynomial combination of its inputs, rather than a linear combination.

Like the median filter, we can interpret a Volterra predictor as an adaptive linear filter. Consider the simple quadratic predictor

$$
P\left(x_{e}[n]\right)=a x_{e}^{2}[n]+b x_{e}[n] x_{e}[n-1]+c x_{e}^{2}[n-1]
$$

where $a, b$, and $c$ are the filter coefficients. Equivalently,

$$
P\left(x_{e}[n]\right)=a^{\prime} x_{e}[n]+b^{\prime} x_{e}[n-1]
$$

where $a^{\prime}=a x_{e}[n]+b x_{e}[n-1]$ and $b^{\prime}=c x_{e}[n-1]$. This shows that any polynomial predictor can be interpreted as a linear filter with data-adaptive coefficients. Thus, we have two options. First, we can perform the update first [7], creating the coarse coefficients from a single update operator prior to applying the nonlinear prediction operator. This ensures the coarse coefficients are a low-pass version of the original data, regardless of predictor. Our second option is to follow an approach similar to that outlined for the median filter to devise the correct update step. In this case, a new update filter must be found for each $c[n]$; these filters will adapt to the Volterra filter coefficients at each point. Thus, the update filters will also be data-dependent.

\section{EXPERIMENTS}

To gauge the quality of our new non-linear DWTs, we compare the performance of the non-linear transforms in signal denoising applications. We also compare the entropies of the coefficient distributions of several well-known test signals to assess the level of compaction afforded by the non-linear transforms.

Denoising. Because DWTs provide such a parsimonious representation of a wide classes of signals, the DWT has proved to be a powerful tool for noise removal. The basic "wavelet denoising" programme [4] is described as follows. We observe $L$ samples $\{x[n]\}$ of an unknown function $f$ with additive i.i.d. Gaussian noise $\{\eta[n]\}$ :

$$
x[n]=s[n]+\eta[n], \quad n=0,1, \ldots, L-1 .
$$

We compute the DWT of $x$ and apply a threshold non-linearity to the wavelet coefficients. The "soft-threshold" sets very small coefficients to zero and reduces all other coefficients by a fixed amount proportional to the standard deviation of the noise. The inverse DWT of the thresholded coefficients produces a "denoised" signal. For more information, see [4].

As seen in Figures 6-8, our median filter DWT performs well for denoising images that are dominated by edges. In general, the median-based lifted transform preserves edges much better than the length-8 Daubechies transform. The median transform suffers from "Haar-like" artifacts, but less so than the actual Haar (Daubechies-2) transform. All three denoising methods illustrated in Figures 6-8 have approximately the same mean-square-error performance.

Compression. By truncating the output of the update filter and removing the $\sqrt{2}$ normalization factors, our algorithm becomes an integer-to-integer transform. This transform (with median update) should lend itself to lossless compression applications. In Table 1 , we compare the weighted entropies of images processed by our non-linear transforms (the first with median predict, the second with both a median predict and a medium update) vs. the lossless transforms of Said and Pearlman [8]. As in [8], these entropies are the weighted means of the entropies in each of the pyramid levels. This is a more accurate estimate of the bit rates if adaptive entropy coding is to be used. The lifted transform with median predict and median update is very competitive. 
Note that our median predict followed by linear update algo rithm cannot be used for compression except when the update order is $\tilde{N}=1$. This is due to the adaptive nature of the median predictor. To preserve the low-pass interpretation of the coarse coefficients, the update filter must adapt based on the median choices. Thus, the prediction choices or the update filters must be stored as side information, which negates any compression improvement realized by the median based transform.

Table 1: First-Order Entropies in Bits Per Pixel

\begin{tabular}{|c|c|c|c|c|c|}
\hline \multirow{2}{*}{ Image } & \multicolumn{5}{|c|}{ Transform } \\
\cline { 2 - 6 } & med $\mathrm{P}$ & med $\mathrm{P}, \mathrm{U}$ & $S+P A$ & $S+P \quad B$ & $S+P C$ \\
\hline camera & 2.86 & $\mathbf{2 . 7 4}$ & 2.92 & 2.93 & 2.94 \\
\hline building & 3.75 & $\mathbf{3 . 7 0}$ & 4.16 & 4.21 & 4.26 \\
\hline bridge & 4.47 & $\mathbf{4 . 3 6}$ & 4.50 & 4.54 & 4.50 \\
\hline
\end{tabular}

\section{CONCLUSIONS}

Our motivation in designing non-linear predictors was to better approximate signals and create more compact signal representations. We employed the lifting construction to incorporate these non-linear predictors and still retain linear properties throughout the transform. In denoising applications, our non-linear predictors show great potential to retain sharp edges while reducing artifacts. Although many of our transforms cannot be used for image compression due to the need for side information, in special cases our non-linear transforms can be used for lossless compression. In these cases, our representations show potential to equal or exceed the performance of existing lossless compression representations.

Future work involves applying other non-linear predictors (such as the Volterra filter) to the denoising and image compression applications. Also, Donoho and Yu have developed median based algorithms in [9] for denoising signals corrupted by heavy-tailed noise. We intend to modify our transforms for these non-Gaussian denoising problems.

\section{REFERENCES}

[1] I. Daubechies, Ten Lectures on Wavelets. New York: SIAM, 1992.

[2] W. Sweldens, "The lifting scheme: A custom-design contruction of biorthogonal wavelets," J. Appl. Comp. Harm. Anal., vol. 3, no. 2, pp. 186-200, 1996.

[3] L. Daubechies and W. Sweldens, "Factoring wavelet transforms into lifting steps," J. Fourier Anal. Appl., vol. 4, no. 3, pp. 245-267, 1998.

[4] D. Donoho, "De-noising by soft-thresholding," IEEE Trans. Inform. Theory, vol. 41, pp. 613-627, May 1995.

[5] F. J. Hampson and J.-C. Pesquet, "A nonlinear subband decomposition with perfect reconstruction," in Proc. ICASSP, 1996.

[6] V. J. Mathews, "Adaptive polynomial filters," IEEE SP Magazine, vol. 8, no. 3, pp. 10-26, 1991.

[7] R. L. Claypoole, G. Davis, W. Sweldens, and R. G. Baraniuk, "Nonlinear wavelet transforms for image coding," in Proc. 31 st Asilomar Conf., (Pacific Grove, CA), 1997.

[8] A. Said and W. Pearlman, "An image multiresolution representation for lossless and lossy compression," IEEE Trans Image Processing, vol. 5, no. 9, pp. 1303-1310, 1996.

[9] D. Donoho and T. Yu, "Robust nonlinear wavelet transform based on median-interpolation," in Proc. 31 st Asilomar Conf., (Pacific Grove, California), pp. 75-79, 1997.

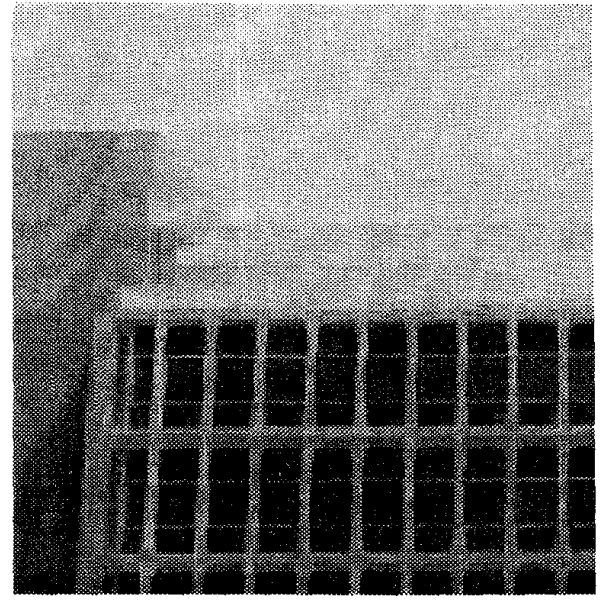

Figure 6: Denoised Image. Median predict, 4 point linear update.

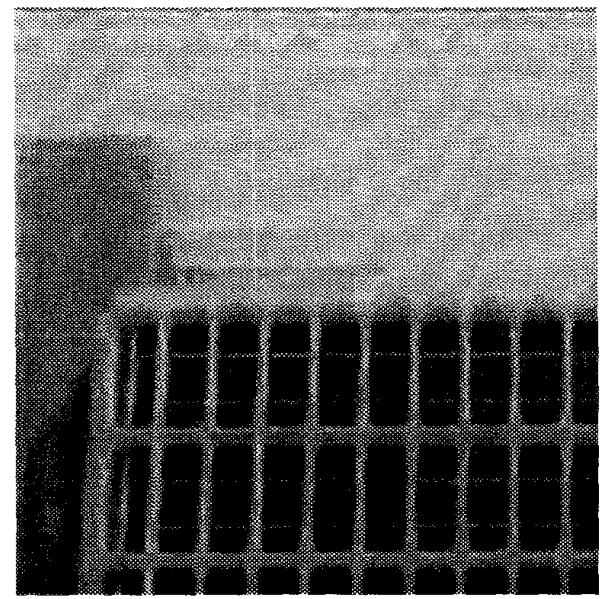

Figure 7: Denoised image using Daubechies-8 DWT.

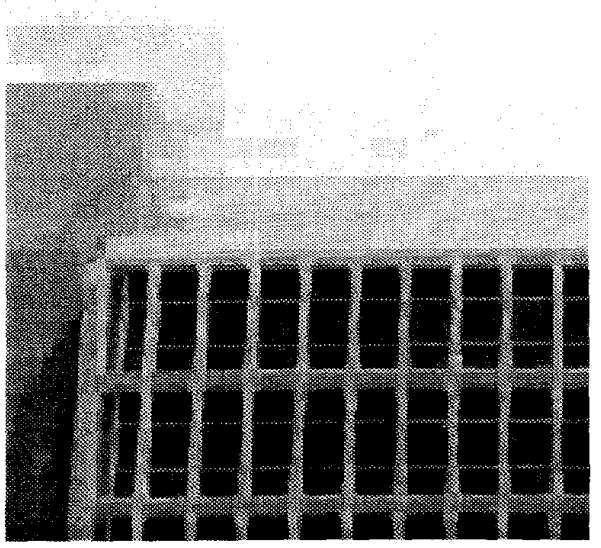

Figure 8: Denoised image using Haar DWT. 